\title{
Estudio económico para la producción y comercialización de hoja de Stevia (Stevia rebaudiana bertoni) deshidratada en Chinú Córdoba
}

\author{
An economic study in Chinú, Cordoba department, for producing \\ and marketing dehydrated Stevia (Stevia rebaudiana bertoni) \\ leaves
}
Estudo económico para a produção e comercialização de folha de stevia (Stevia rebaudiana bertoni) desidratada, Chinú-Córdoba

Euriel Millán - Romero ${ }^{1 *}$, Wilson Pacheco - Díaz ${ }^{2 *}$, Jorge Morales - Alemán ${ }^{3 *}$

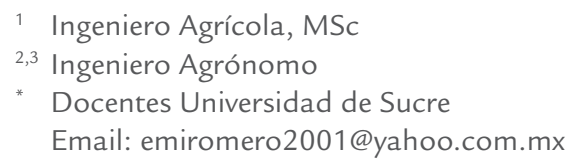

Recibido: mayo 10 de 2011

Aceptado: mayo 28 de 2012

\section{Resumen}

Se estructuró un plan de negocios que propone el uso de la Stevia (Stevia rebaudiana bertoni con fines de comercialización de la hoja seca y como alimento para bovinos. Se realizo encuestas y entrevistas con los productores, empresas transformadoras y consumidores de productos agropecuarios para obtener información de la demanda y la oferta. Para los años 2009, 2010 y 2011 la demanda para la hoja seca fue $944.400 \mathrm{~kg}, 1.065 .600 \mathrm{~kg}$ y $1.880 .800 \mathrm{~kg}$ respectivamente, mientras como alimento para los años 2011, 2012 y 2013 fue 1.377 bultos, 1.605 bultos y 1994 bultos respectivamente. En contraste, la oferta fue de $78.300 \mathrm{~kg}, 107.210 \mathrm{~kg}$ y $149.098 .2 \mathrm{~kg}$ de hoja seca, y como alimento de 3.599 bultos, 3.810 bultos y 3.740 bultos para los años anteriormente citados.

La tasa interna de retorno (TIR) fue 35.29\% con VPN de $\$ 59.627 .458$ a una tasa de oportunidad de $18 \%$ y una relación costo beneficio $\mathrm{RC} / \mathrm{B}=1.57$ lo que indica que el plan de negocio es rentable. La producción de estévia promete ingresos superiores a los de los cultivos tradicionales, producción constante, tecnificación rural y mejoramiento de la calidad de vida de los campesinos. Se concluye que es un plan de negocio atractivo para su implementación, debido a que es un producto rentable, de alto rendimiento y de excelente venta.

Palabras claves: Stevia rebaudiana bertoni, rentabilidad, estudio de mercado, plan de negocio.

\begin{abstract}
A business plan was structured which proposed using Stevia (Stevia rebaudiana bertoni) for marketing dry leaves and using them as food for cattle. Surveys and interviews were held with agricultural product producers, companies and consumers to
\end{abstract}


obtain relevant supply and demand data. Dried leaf demand was 944,400 kg, 1,065,600 kg and 1,880,800 kg in 2009, 2010 and 2011, respectively, and demand for sacks of leaf as food was 1,377, 1,605 and 1,994 for 2011, 2012 and 2013 , respectively. By contrast, supply was 78,300 kg, 107,210 kg and 149,098.2 kg dry leaf and 3,599, 3,810 and 3,740 sacks of leaf as food for the aforementioned years. The internal rate of return (IRR) was 35.29\%, net present value (NPV)\$59,627,458with $18 \%$ opportunity rate and 1.57 cost benefit ratio, thereby indicating that the business plan was profitable. Stevia production would seem to offer income above that for traditional crops, steady production, rural modernisation and an improvement infarmers' quality of life. It may thus be concluded that implementing the aforementioned business plan is attractive as it deals with a profitable product having high yield and excellent sales' potential.

Key words: Stevia rebaudiana bertoni, profitability, market research, business plan.

\begin{abstract}
Resumo
Foi estruturado um plano de negócios que propõe o uso de Stevia (Steviar ebaudiana bertoni) com a finalidade de comercializar a folha seca e também como alimento para o gado. Realizaram-se enquetes e entrevistas com produtores, empresas processadoras e consumidores de produtos agrícolas e pecuários para obter informação a oferta e a procura. Para os anos 2009, 2010 e 2011, a procura por folhas secas foi $944.400 \mathrm{~kg}, 1.065 .600 \mathrm{~kg}$ e $1.880 .800 \mathrm{~kg}$, respectivamente, e como alimento para os anos 2011, 2012 e 2013 foram de 1.377, 1.605 y 1.994 sacos de produto, respectivamente. Em contraste, a oferta foi de $78.300 \mathrm{~kg}, 107.210 \mathrm{~kg}$ e $149.098 .2 \mathrm{~kg}$ de folhas secas, e de alimentos $3.599,3.810$ e 3.740 sacos para os anos acima mencionados.

A taxa interna de retorno (TIR) foi de 35,29\% com VPN de \$59.627.458 com uma taxa de oportunidade de18\%, e uma relação de custo-benefício $\mathrm{RC} / \mathrm{B}=1,57$ indicando que o plano de negócio é rentável. A produção de stevia promete renda superior às culturas tradicionais, produção constante, modernização rural e melhora na qualidade de vida dos camponeses. Conclui-se que o plano de negócio é atraente para sua implementação, devido ao fato, que é um produto rentável, de elevado rendimento e venda excelente.
\end{abstract}

Palavras chave: Stevia rebaudiana bertoni, rentabilidade, pesquisa de mercado, plano de negócios.

\section{Introducción}

La industria de la stevia es un mercado creciente a nivel mundial, países desarrollados como Japón e Israel la utilizan desde hace más de 40 años. El nivel de consumo en el Japón alcanza el $60 \%$ de la población total, lo que ha motivando a grandes empresas como Coca Cola a utilizarla para su comercialización en este atractivo mercado. Egipto, Gran Bretaña, Arabia Saudita e Israel entre otros, han entrado en la era de la stevia, desarrollando productos y adoptando la cultura del endulzante natural, que además, de sus usos medicinales y alimenticios tiene otras propiedades de interés para la industria cosmética (López y Peña, 2004).

La stevia es conocida como hoja dulce, su extracto, contiene hasta 300 veces más la dulzura del azúcar. La creciente demanda por productos bajos en carbohidratos y bajos en azúcar han visto en la stevia un alimento alternativo (Marín, 2004). La stevia se ha utilizado en el tratamiento de la obesidad y la hipertensión arterial, mejorando así la calidad de vida de las personas que padecen estas enfermedades, además de otros beneficios (Mejía, 2009).

En Colombia el consumo de endulzantes con base en estévia alcanza el $10 \%$ del mercado nacional, contra un $4 \%$ del mercado mundial (excluido USA) y un $1 \%$ del mercado de USA. De hecho, el conocimiento de la estévia del consumidor colombiano es muy superior al promedio latinoamericano (excluido Brasil), por eso, actualmente se encuentran endulzantes con base en stevia en supermercados, tiendas naturistas, restaurantes, hoteles y cafeterías donde se ofrecen como una opción para endulzar comidas y bebidas (Barriocanal, 2009)

Se estima que entre las varias marcas que hay en el mercado, se consumen unos tres millones mensuales de porciones de steviosido de $1.0 \mathrm{~g}$, lo que equivale a una utilización de 250 kilos de steviosido y un número aproximado de 30.000 consumidores mensuales (López y Peña 2004).

El objetivo de este estudio fue estructurar un plan de negocio que permitiese evaluar la posible comercialización de la hoja de estevia como edulcorante y como alimento para el ganado bovino en la vereda Palmital del municipio de chinú (córdoba).

\section{Materiales y metodos}

El presente estudio se realizo en la finca San José, vereda Palmital del municipio de Chinú, Córdoba, distante $15 \mathrm{~km}$ del casco urbano al noroeste del mismo. A una altura de $100 \mathrm{msnm}$, precipitación anual de $1727 \mathrm{~mm}$ (IDEAM, 2010) y temperatura media de $28^{\circ} \mathrm{C}$, bajo las coordenadas ( $9^{\circ} 7^{\prime} 0^{\prime \prime}$ Norte, $75^{\circ} 24^{\prime} 0^{\prime \prime}$ Oeste). La zona presentaba buenas vías de comunicación, suelos 
con alta potencialidad agrícola, buena oferta de mano de obra y fácil acceso a los proveedores. La especie utilizada fue la Stevia rebaudiana bertoni var. morita II.

Se aplicó una encuesta a 10 productores para obtener información cuantitativa sobre la cantidad de producto obtenido por hectárea, la densidad de las plantas, los sistemas de producción y rendimiento. Se obtuvo además información cualitativa sobre la tendencia del mercado y la rentabilidad. Se realizo otra encuesta a empresas transformadoras de la hoja seca de stevia ubicadas en la ciudad de Medellín (Antioquia), para obtener información sobre cantidad de producto transformado y el requerido para trabajar a capacidad total. Otra tercera encuesta se aplicó a los almacenes agropecuarios y finqueros en donde se indagó por la cantidad de productos de alimento para bovinos vendidos por año y la posibilidad de que se adoptase la stevia como alimento para los bovinos. Toda la información se obtuvo para los años 2009, 2010 y 2011.

De acuerdo a la información anterior y basándose en el paquete tecnológico de producción de la estévia que en este proyecto se propone, se fija una cantidad de producción de stevia de $1.800 \mathrm{~kg}$ mensuales y 240 bultos (50 kg por bulto) de alimento para bovinos. De igual manera se obtuvo el punto de equilibrio en la producción anual durante 5 años.

Se determinó una producción de 1.800 kg de hoja seca de stevia mensuales con una densidad de siembra de 100.000 plantas por hectárea, aplicando el paquete tecnológico para la producción de stevia propuesto por Agrobiológicos Safer. Este último ofrece un paquete de producción bastante favorable para la producción de stevia que permite obtener producciones hasta de $2.000 \mathrm{~kg} / \mathrm{ha}$.

La rentabilidad del proyecto fue evaluada mediante un análisis financiero que relacionan los flujos de entrada por un periodo de 5 años en los cuales se proyecta la duración del plan de negocio. Para el cálculo de estas variables se utilizo el formato financiero otorgado por el fondo emprender para la evaluación de proyectos. Las variables analizadas fueron valor presente neto (VPN), tasa interna de retorno (TIR), periodo de recuperación de la inversión (PRI), relación costo beneficio (RC/B), (Baca, 1989).

Se tomaron de igual manera consideraciones y afirmaciones de productores, empresarios y consumidores de alimento para bovinos referenciados en las condiciones del mercado de la stevia, las tendencias de este y la rentabilidad de la producción.

\section{Evaluación económica}

Para el cálculo de los indicadores, se utilizó las siguientes formulas matemáticas.

Valor actual neto (VAN):

$$
V A N=\sum_{t=1}^{n} \frac{V_{t}}{(1+k)^{t}}-I_{0}
$$

Donde:

$t=$ tiempo
$n=$ años de vida útil del negocio
$V=$ Flujo neto de entrada en $t$
$K=$ tasa de interés.
$I_{0}=$ Inversión Inicial

Tasa interna de retorno (TIR):

$$
T I R=\frac{-1+\sum_{i=1}^{n} F_{i}}{\sum_{i=1}^{n} i * F_{i}}
$$

Donde:

$\mathrm{I}=$ inversión inicial

$\mathrm{Fi}=$ flujo de caja del proyecto (ingresos menos egresos)

$\mathrm{i}=$ tiempo

$\mathrm{n}=$ vida útil del proyecto

Relación beneficio costo (RB/C)

$$
\frac{R B}{C}=\frac{V P i}{V P \in}
$$

Donde:

VPe $=$ Valor Presente de los egresos.

$\mathrm{VPi}=$ Valor Presente de los ingresos.

Periodo de recuperacion de la inversion (PRI)

$$
P R I=\frac{\text { Inversión inicial }}{\text { Utilidades promedio }}
$$

Rentabilidad obtenida en la empresa:

$$
R O E=\frac{\text { Beneficio neto }}{\text { Patrimonio neto }}
$$

\section{Resultados}

La demanda insatisfecha de la hoja de stevia seca se muestra en la tabla 1. La información arrojo resultados favorables para la comercialización de este producto en donde el $100 \%$ de los encuestados calificaron el cultivo como altamente rentable, pero los altos costos iniciales y el mantenimiento limitan su producción. Los encuestados aseguraron tener un rendimiento de 1.500 a $1.800 \mathrm{~kg} / \mathrm{ha}$, sin embargo si se utilizara el pa- 
quete tecnológico que se plantea (Pamies, 2008) podrían lograr un rendimiento hasta de $2.000 \mathrm{~kg} / \mathrm{ha}$. Por otra parte, los productores manifiestan que las empresas compradoras les solicitan más producto, pero que debido al área sembrada actualmente, no pueden cumplir con este requerimiento.

En la tabla 1, se resumen las cantidades en kilogramos de compra de hoja seca de stevia que cada una de las empresas encuestadas compra actualmente; al igual que la cantidad de producto que los productores de la zona están vendiendo en este momento, se observa una cantidad de $958.390 \mathrm{~kg}$ como demanda insatisfecha para el año 2012, lo cual ofrece expectativas en cuanto a la venta de la hoja seca. En opinión de compradores y productores, la stevia y sus productos derivados, va en constante crecimiento ya que existe la tendencia al consumo de productos bajos en calorías por parte de las personas preocupadas por su salud.

Tabla 1. Demanda insatisfecha de hoja de stevia $(\mathrm{kg})$

\begin{tabular}{l|c|c|c}
\hline \multicolumn{1}{c|}{ Descripcion } & $\mathbf{2 0 0 9}$ Año & $\mathbf{2 0 1 1}$ \\
\cline { 2 - 4 } & $\mathbf{2 0 1 0}$ & 1.880 .800 \\
\hline $\begin{array}{l}\text { Total demanda (5 Empresas } \\
\text { compradoras) en Kg. }\end{array}$ & 944.400 & 1.065 .600 & 149.098 .2 \\
$\begin{array}{l}\text { Total oferta (10 productores } \\
\text { cercanos a la zona) en Kg }\end{array}$ & 78.300 & 107.210 & 1.731 .702 \\
\hline
\end{tabular}

En la tabla 2 se muestra la demanda actual de productos de alimento para bovinos entre los cuales están sales mineralizadas, concentrados, melazas y forraje, este último más que en almacenes agropecuarios se encuentra en fincas productoras de forraje al igual que los bloques nutricionales. Los resultados de la encuesta permitió identificar que en la región no se comercializa alimentos para bovinos con base en la stevia, por lo que se debe realizar un plan de mercadeo activo para promocionar el producto. El estudio de mercado en donde se indago por la compra de este nuevo producto, mostró que el $53.33 \%$ de la población (finqueros y almacenes agropecuarios), está dispuesta a reemplazar el alimento animal tradicional por el extraído de la planta de stevia, considerando los beneficios que este ofrece, enfatizando de esta manera que el nuevo producto tiene gran aceptación en el mercado local, y un $13.3 \%$ tienen incertidumbre de cambiarlo, pero que con insistencia sobre las bondades y la competencia en los costos del producto, se podría llegar a su convencimiento.

En la tabla 2, se observa que la demanda de la stevia, como alimento para bovinos es bastante grande a pesar de ser un producto nuevo en la región. Se puede afirmar que en corto plazo y dependiendo de los resultados obtenidos en los animales con este nuevo producto, se incrementaría el consumo del mismo, obteniendo un alto posicionamiento en el mercado agropecuario.

Los resultados de la encuesta a los almacenes agropecuarios aseguraron vender en promedio esta cantidad de productos anuales, dentro de los cuales se identificaron melaza, concentrado comercial, salvado de arroz y maíz, bloques nutricionales y pacas de forrajes.

En la tabla 3. Se presenta la cantidad de hoja deshidratada como de alimento para bovinos. De acuerdo con las consultas realizadas dentro del estudio de mercado a la cámara de comercio, las perdidas en la producción de stévia (castecol) en el país, tiene una variación anual del 10\%. Con respecto a la producción normal de $2000 \mathrm{~kg} / \mathrm{ha}$ con corte cada dos meses (Mejía, 2009), en el proyecto se asigno un porcentaje de $10 \%$ para obtener la estimación de la capacidad de acuerdo a la demanda y la variación en ventas. Respecto a la producción de alimento para bovinos se tomo una producción con una capacidad máxima instalada igual a la capacidad utilizada. Se puede ver que entre el año 1 y 2 hay un cambio en la producción de más del $100 \%$ esto en razón a que durante el primer año existen 8 meses de adecuación y etapa improductiva del proyecto, por lo tanto, solo se producirán los últimos 5 meses del primer año. 
Tabla 2. Demanda de alimento para bovinos en el municipio de Chinú (Cordobá).

\begin{tabular}{l|c|c|c}
\hline \multirow{2}{*}{ Descripción } & \multicolumn{3}{|c}{ Demanda de bultos anuales } \\
\cline { 2 - 4 } & $\mathbf{2 0 1 1}$ & $\mathbf{2 0 1 2}$ & $\mathbf{2 0 1 3}$ \\
\hline $\begin{array}{l}\text { Ventas anuales de productos similares } \\
\begin{array}{l}\text { Demanda anual de alimento para } \\
\text { bovinos a base de estevia }\end{array}\end{array}$ & 3599 & 3810 & 3740 \\
\hline
\end{tabular}

Esta proyección se obtuvo teniendo en cuenta el promedio de producción de hoja de stevia seca que obtienen los productores aplicando el paquete tecnológico que se pretende utilizar en este proyecto. Los promedios de producción están en un rango de 1.800 a $2.000 \mathrm{~kg}$ de hoja seca/ha. Además se redujo un porcentaje de $10 \%$ de la producción total, establecidos como perdidas en el producto por diversos factores; este porcentaje se asigno con base en las experiencias de los productores. La cantidad de producto de alimento para bovinos se obtuvo a partir de la cantidad de residuos de cosecha que se obtienen de las tres hectáreas planteadas por el proyecto.

Se puede inferir con base en esta producción y la menor área del cultivo que es un cultivo que presenta bondades obteniendo altas producciones en pequeñas áreas. La producción que se muestra en la tabla 3 se obtuvo mediante un paquete tecnológico especializado para la producción de stevia, de igual manera la cantidad de producción de alimento para bovinos.

Los gastos de inversión y funcionamiento para poner en marcha el plan de negocio se muestran en la tabla 4.

Tabla 3. Proyección de producción de hoja seca y alimento para bovinos.

\begin{tabular}{c|c|c}
\hline Años & $\begin{array}{c}\text { Estimación de Producción/ } \\
\text { Año }(\mathbf{k g})\end{array}$ & Demanda (Bultos 50Kg) \\
\hline 1 & 9000 & 240 \\
2 & 23850 & 768 \\
3 & 29250 & 912 \\
4 & 34650 & 1056 \\
5 & 40050 & 1200 \\
\hline
\end{tabular}

Tabla 4. Inversiones requeridas para poner en marcha el plan de negocio.

\begin{tabular}{|c|c|c|c|c|c|c|}
\hline \multirow{2}{*}{ Descripción } & \multicolumn{6}{|c|}{ Año } \\
\hline & $\mathbf{0}$ & 1 & 2 & 3 & 4 & 5 \\
\hline Costos de inicio & $570,000.00$ & & & & & \\
\hline Costos preliminares & $1,989,000.00$ & & & & & \\
\hline Materia prima & $30,000,000.00$ & & & & & \\
\hline $\begin{array}{l}\text { Maquina, equipos } \\
\text { y herramientas }\end{array}$ & $14,281,000.00$ & & & & & \\
\hline Mezcla de mercadeo & & $1,433,460$ & $2,289,650$ & $2,658,075$ & $3,056,336$ & $3,486,581$ \\
\hline Gastos de Administración & & $11,584,800$ & $22,800,000$ & $23,712,000$ & $24,660,480$ & $25,646,899$ \\
\hline Gastos administrativos & & $4,223,500$ & $4,392,440$ & $4,567,137$ & $4,749,704$ & $4,939,255$ \\
\hline
\end{tabular}




\section{Costo total de producción}

Los costos totales de producción (Figura 1), presentaron un incremento constante anualmente, debido a que los precios de los productos estaban sujetos a la inflación, la cual se tuvo en cuenta un valor de $3 \%$, también a que la producción de la empresa iba en constante crecimiento con el tiempo.

Los costos variables para la producción de las tres hectáreas iniciales de stevia con las cuales se establecerá la empresa con una densidad de 100.000 plantas/ha se muestran en la figura 2.

\section{Ingresos y Punto de equilibrio}

Los ingresos esperados de hoja deshidratada de stevia se presentan en la figura 3, para el primer año fueron de $\$ 81.000 .000$ para una producción de $9.000 \mathrm{~kg}$. En la figura 4 se observa el punto de equilibrio que fue de $8198.3 \mathrm{~kg}$ para un monto total de $\$ 73.784 .735$. En la figura 5 se muestra el punto de equilibrio para el primer año el cual correspondió a 106.28 bultos por un valor de \$ 850.246. Los ingresos fueron calculados con base al sistema de producción mensual de la empresa tanto para la hoja seca de stevia como para el alimento de bovinos, obteniendo una producción lineal anual.

Los precios de ventas utilizados fueron los vigentes por los que se comercializaba la hoja seca en el mercado de Medellín-Colombia. Se estableció el precio del alimento para bovinos, teniendo en cuenta los costos de producción utilizados para obtener un bulto de 50 kg de producto, basado en los siguientes parámetros: punto de equilibrio, costos de materia prima, costos de manos de obra y ganancias anticipadas.

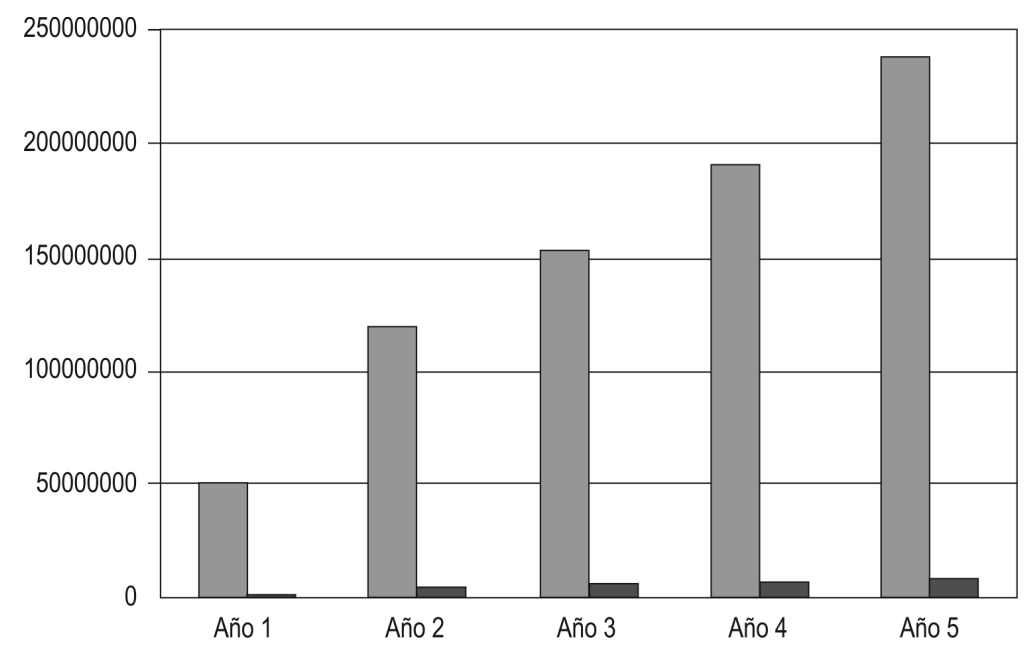

Costos de producción de hoja deshidratada de estévia

Costos de producción de alimento para bovino

Figura 1. Costos totales de producción de hoja deshidratada de stevia y alimento para bovinos.

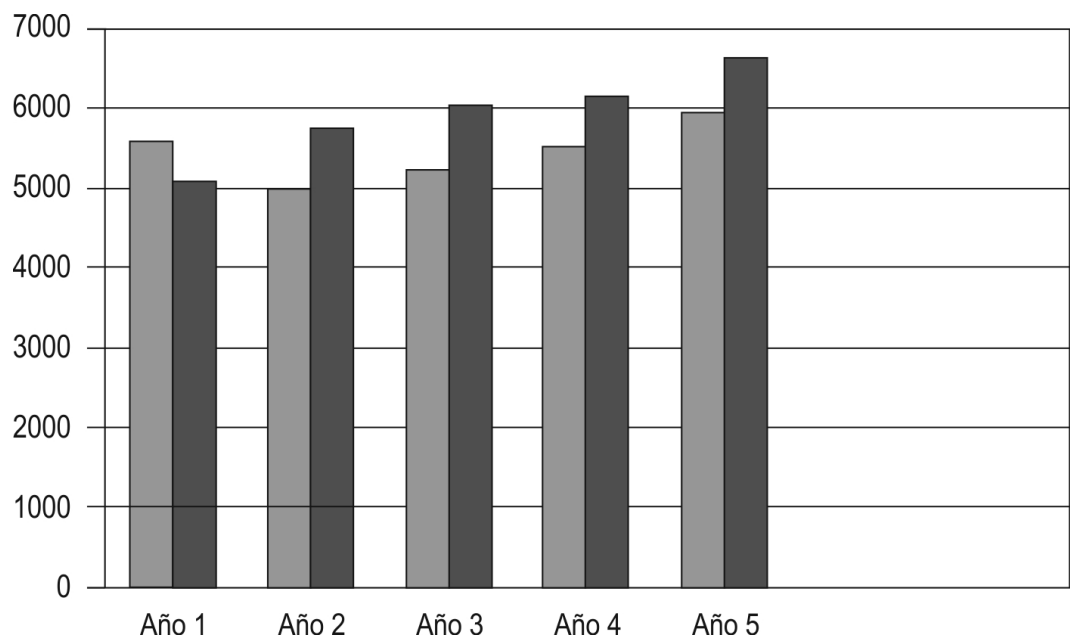

Costos variables hoja seca de estévia

Costos variables alimento para bovinos

Figura 2. Costos variables de producción de hoja deshidratada y alimento bovino 


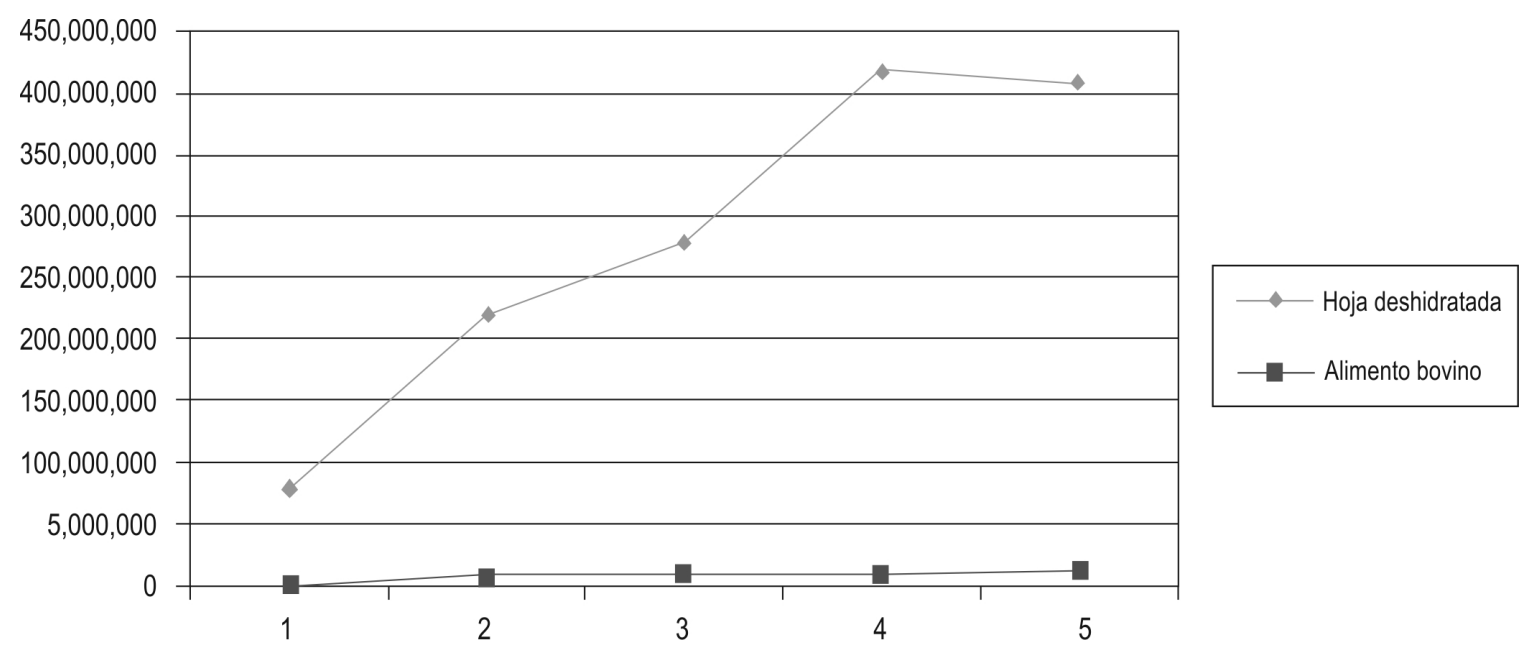

Figura 3. Ingresos esperados para hoja deshidratada y alimento bovino (en pesos)

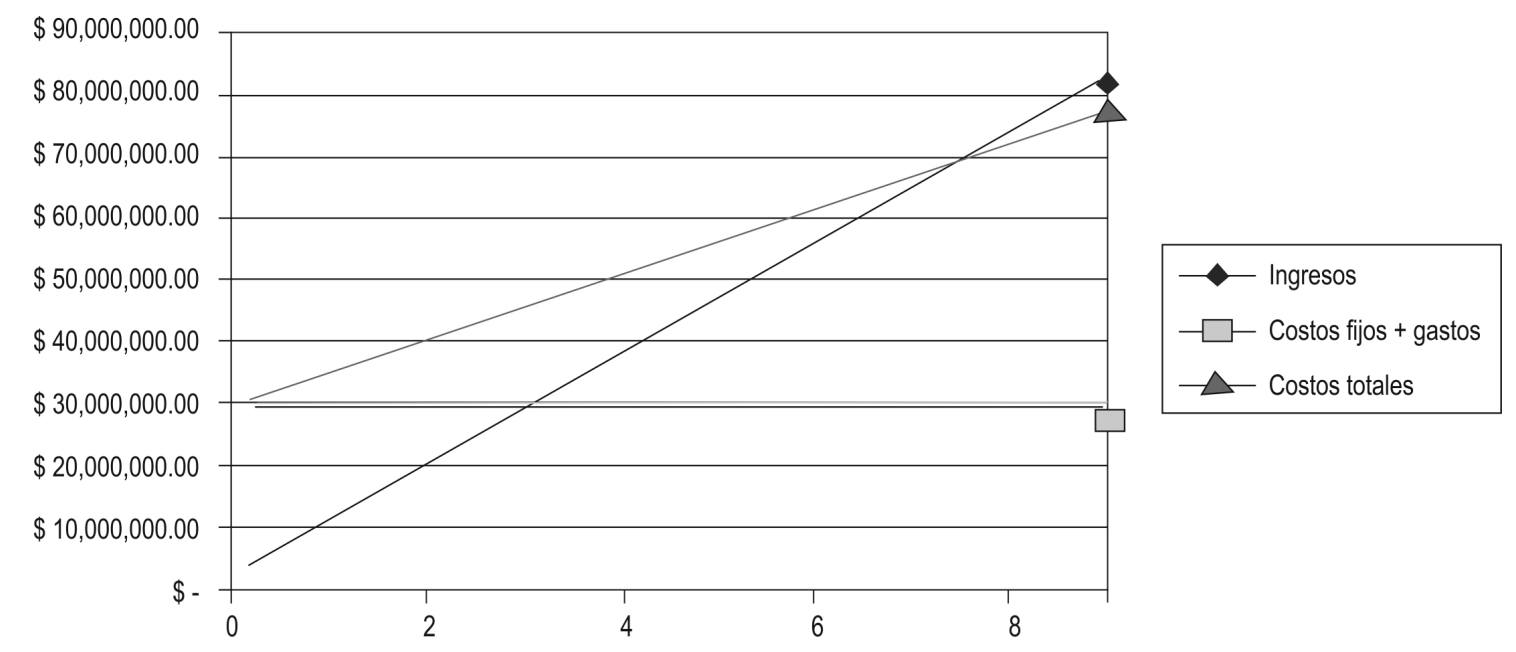

Figura 4. Punto de equilibrio año1, hoja deshidratada de stévia

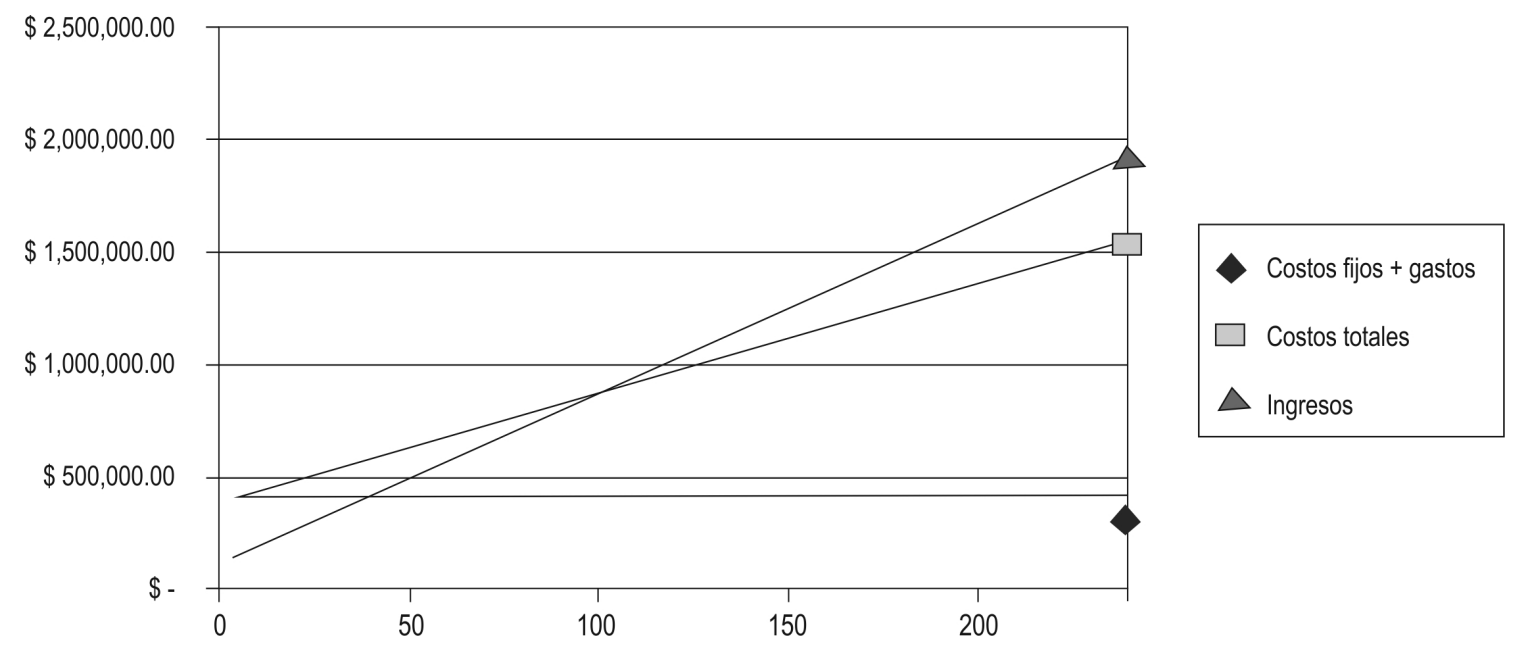

Figura 5. Punto de equilibrio año1, alimento bovino 


\section{Índices de evaluación económica}

La tasa de oportunidad adoptada fue de un $18 \%$, generalmente utilizada por el fondo emprender para evaluar los planes de negocios, de lo anterior, se obtuvo un $\mathrm{VPN}=\$ 59.627 .458$, que sugiere que el proyecto es financieramente atractivo, ya que el dinero invertido produce una rentabilidad superior a la tasa de oportunidad empleada. Además, la $\mathrm{TIR}=35.29 \%$ y una relación costo beneficio de 1.57 indicando que el proyecto sobrepasa la tasa de oportunidad empleada. El periodo de recuperación de inversión PRI fue 1.76 y la rentabilidad de $31 \%$.

\section{Discusión}

Con las variables estudiadas se puede concluir que el proyecto es altamente rentable con una tasa de $31 \%$ y que de acuerdo con los objetivos propuestos, es muy favorable para la producción en la zona objeto de estudio. Respecto a los niveles de producción derivados de la información obtenida con los productores y los realizados por CORPOICA y la Universidad de Córdoba, dan cuenta que la zona presenta buenas condiciones para la producción de Stevia rebaudiana bertoni variedad morita II.

Investigaciones relacionadas con el área foliar de la variedad morita II fueron superiores a la variedad morita I lo que sugiere que la primera posee mejores características morfo-fisiológicas y genéticas que le permiten ser más eficiente. Los altos niveles de radiación inducen un incremento de la materia seca de las hojas y materia seca total en morita II, efecto que no se evidencio para morita I, (Jarma et al., 2005).

Estudios realizados con anterioridad en Córdoba identifican la potencialidad de este departamento como gran productor de stevia, dadas las condiciones ambientales favorables entre ellas, los suelos y la oferta de luz diaria (Espitia et al., 2009).

Las investigaciones adelantadas por la Universidad de Córdoba, (datos sin publicar), aunque no todas relacionadas en este articulo, pueden considerarse como un primer paso en la identificación de tecnologías competitivas, que hagan de la agroindustria de la stevia un negocio rentable y a su vez genere bienestar social en la región.

En las anteriores investigaciones se obtuvieron producciones de materia seca por hectárea alrededor de $2.300 \mathrm{~kg}$ suministrando fertilización y todos los requerimientos necesarios para el desarrollo de la planta. (Espitia et al., 2009).
El uso de la stevia en la alimentación animal no se ha explorado por completo, aunque se viene promoviendo su uso con tal propósito; algunas investigaciones se han orientado a su aplicación como saborizante de pastos (en animales de granja y domésticos), con el subsecuente aumento en la producción. Se cree además que estimula el apetito e interviene en la pronta recuperación después de procesos infecciosos, mejora el sabor y la calidad de la carne (menor exudación y mejor conservación), disminuye la cantidad de huevos rotos en ponedoras y mejora la calidad de la carne de pollo. Subsecuentemente previene la erosión y ulceración de la molleja en aves, reduce la mortalidad en empresas piscícolas, produciendo pescado más sano, el cual se mantiene fresco por más tiempo (Jarma, 2008).

En conclusión el proyecto es una alternativa viable y rentable para crear desarrollo rural, por tanto, se recomienda para su ejecución en la zona objeto con el fin de mejorar la calidad de vida de los campesinos.

\section{Referencias}

Baca G. Ingeniería Economica.1989. Politécnico Gran colombiano. Bogotá DC Colombia., Octava ed. p- 413.

Barriocanal L. Área Web revista alimentos; stevia, una alternativa para los que no deben consumir azúcar, junio 10, 2009.

Espitia M, Montoya R, Atencio L. Rendimiento de Stevia rebaudiana Bert. Bajo tres arreglos poblacionales en el Sinú medio. Revista U.D.C.A. Actualidad \& Divulgación Científica 2009,12(1):151161.

IDEAM. Instituto de Hidrología y Meteorología y Estudios Ambientales. Datos meteorológicos, 2010.

Jarma A, Rengifo T, Araméndiz H. Aspectos fisiológicos de stévia (Stevia rebaudiana Bertoni) en el Caribe colombiano: I. Efecto de la radiación incidente sobre el área foliar y la distribución de biomasa. Agronomía. Colombiana 2005;23(2):207-216.

Jarma A. Estudios de adaptación y manejo integrado de estévia (Stevia rebaudiana Bert.): nueva alternativa agroindustrial del Caribe colombiano. Revista Colombiana De Ciencias Hortícolas 2008;2(1):110-121.

López L, Peña L. 2004. Plan estratégico para la creación de una empresa dedicada a la producción y comercialización de edulcorante a base de stevia. Universidad Javeriana. Diciembre. P-125

Marín W. 2004. Sondeo de mercado de la estévia. Instituto de Investigación de Recursos Biológicos Alexander Von Humboldt.

Mejía F. (asesor Comercial) COOPRODESTEVIA-Desarrollo económico de la stevia para comunidades y futuras empresas- Cultivos orgánicos Barbosa Antioquia Colombia. (www.cooprodestevia. blogspot.com). 2009.

Pamies J. Manual del cultivo de la estévia. Blog post http://joseppamies.wordpress.com. 04, 2008 